\title{
A Framework to Support Ubiquitous Healthcare Monitoring and Diagnostic for Sickle Cell Disease
}

\author{
Mohammed Khalaf ${ }^{1}$, Abir Jaafar Hussain ${ }^{1}$, Dhiya Al-Jumeily ${ }^{1}$, Paul Fergus ${ }^{1}$ \\ Russell Keenan ${ }^{2}$ and Naeem Radi ${ }^{3}$ \\ ${ }^{1}$ Applied Computing Research Group, School of Computing and Mathematical Sciences, \\ Liverpool John Moores University, Byrom Street, Liverpool, L3 3AF, UK \\ ${ }^{2}$ Liverpool Paediatric Haemophilia Centre, Haematology Treatment Centre, Alder Hey \\ Children's Hospital, Eaton Road, West Derby, Liverpool L12 2AP, UK \\ ${ }^{3} \mathrm{Al}$ Khawarizmi International College, Abu Dhabi, United Arab Emirates \\ M.I.Khalaf@2014.ljmu.ac.uk, \{a.hussain, d.aljumeily, P.Fergus\}@ljmu.ac.uk, \\ Russell.keenan@alderhey.nhs.uk, n.radi@khawarizmi.com
}

\begin{abstract}
Recent technology advances based on smart devices have improved the medical facilities and become increasingly popular in association with realtime health monitoring and remote/personals health-care. Healthcare organisations are still required to pay more attention for some improvements in terms of cost-effectiveness and maintaining efficiency, and avoid patients to take admission at hospital. Sickle cell disease (SCD) is one of the most challenges chronic obtrusive disease that facing healthcare, affects a large numbers of people from early childhood. Currently, the vast majority of hospitals and healthcare sectors are using manual approach that depends completely on patient input, which can be slowly analysed, time consuming and stressful. This work proposes an alert system that could send instant information to the doctors once detects serious condition from the collected data of the patient. In addition, this work offers a system that can analyse datasets automatically in order to reduce error rate. A machine-learning algorithm was applied to perform the classification process. Two experiments were conducted to classify SCD patients from normal patients using machine learning algorithm in which 99\% classification accuracy was achieved using the Instance-based learning algorithm.
\end{abstract}

Keywords: Sickle Cell Disease, Mobile Healthcare Service, Real-time data, Self-care Management System, e-Health

\section{Introduction}

Nowadays, the development in communication technologies and their implementation in medical/science data have helped the utilisation of new solutions to enhance healthcare services and outcomes. SCD is one of the most prevalent diseases, which could have an influence on patients' lives due to red blood cell (RBC) abnormality. SCD is caused by a genetic disorder that reduces life expectancy around 45years in developed countries but it is rare for children with sickle cell in Africa and Asia 
continents to survive beyond their $5^{\text {th }}$ birthday. The main reason behind this disease is that a group of ancestral disorders have an impact on the protein inside the RBC called haemoglobin. SCD can easily inherited to the child throughout genetic of sickle haemoglobin ( $\mathrm{Hb} \mathrm{S}$ ) either from both parents or from one of them measured as abnormal haemoglobin [1].

According to the World Health Organisation (WHO), 7 million born each year suffer either from congenital anomaly or from an inherited disease [2]. Furthermore, $5 \%$ of the population around the globe carries traits genes for haemoglobin disorders, primarily, thalassemia and SCD [3]. Within this context, there is still a significant need for constructing cooperative care environment to improve quality and safety of care, and increase caregivers' efficiency with the purpose of providing regular information for association healthcare providers and patients. In order to provide such facilities, this research focuses on the development of self-care monitoring system (personalised monitoring system) based on mobile devices. Therefore, continuous self-care monitoring of chronic diseases and medicine intake are vital for patients to mitigate the severity of the disease by taking the appropriate medicine at the proper time.

Over the past decade, mobile technologies have supported medical fields through intelligent data analytics. The state of the arts growth of communication technologies has raised the capacity to adopt smart devices health applications in clinics and medical environments [4]. The quick improvement of e-Health systems concentrate on developing the pattern of diagnosing as well as treating chronic diseases with the assistance of disease management centre or integrated care strategies [5]. The main reason behind using mobile devises is that they can offer real-time healthcare services efficiently, delivering information and more flexible tools for doctors-patients interaction, which is relevant to the patient's condition. They are also assist healthcare professionals in order to give accurate decision-making and store data for further investigation. These developments bring potential advantages to both medical expert's staff and patients; doctors can concentrate more on high priority responsibilities and patients could acquire a regular consultancy without having regular visits to hospitals, especially if they live in a remote area [6].

This paper provides a framework for monitoring and managements of patients' diary to provide better health-care and services for patients who suffer from SCD. An initial essential part of the proposed system is a machine learning algorithm which is used to classify patients who suffer from the disease and those who do not.

The remainder of this paper is organised as follows. Section 2 will discuss literature review about SCD while section 3 shows the proposed monitoring system. The methodology for classifying sickle cell patients from normal patients is illustrated in section 4, while section 5 shows the conclusion section. 


\section{Related Work}

Intelligent mobile medical care plays an important tool on providing accurate information for immediate healthcare services and diagnosis. Self-care management system provides patients and medical experts continuous monitoring to obtain instant response and save people lives in case of abnormal conditions. In recent years, healthcare organisations worldwide have faced many problems in order to meet the demands of enhanced medical sectors. There are a number of research projects developed for healthcare environments based on mobile computing. Tabish et al [7] proposed a ubiquitous healthcare solution based on IPv6 over Low power Wireless Personal Area Networks (6LowPAN) that uses sensors to record electrocardiogram (ECG) and temperature data to a remote processing centre. This solution, named as Uhealth uses $3 \mathrm{G} / 4 \mathrm{G}$ communication to provide real-time monitoring to the patients within a mile of coverage area.

Kim et al [8] developed a new framework based on ubiquitous healthcare systems, which can work anywhere and at any time. In this case, their system provides realtime service based upon various biosensors measurements such as Electrocardiogram (ECG), blood pressure and temperature. They also have created Hadoop platform (Big Data Centre) in order to store medical data. Leijdekkers [9] proposed a new prototype system supported by wireless sensor network (biosensor) and smart phone to monitor heart fluctuation. The system itself provides analysis of the giving data and automatically alarms the ambulance to take action immediately. Furthermore, the system transmits sensor data to a healthcare management centre for further investigations. Chen et al [10] presented pervasive healthcare monitoring system (PHMS) combined with cloud computing environment, mobile application, and planar super wideband (SWB). The PHMSs can deliver facilities for those who need long term and continuous collection of data, in particular disabled and elderly people for living independent life. The vital signs monitor (VSM) can deliver immediate information to the healthcare centre server with regard to analysis and storage of such data. The medical experts can seamlessly access the database and check final status.

A mobile phone diary platform for adolescent in association with SCD is proposed [11]. This solution can provide principal support for the utilisation of electronic diary to track symptoms and pain for young people. Their work based on utilising the internet communication capability of state-of-the-art smartphone based on 3G/4G [12]. Jung, et al [13] established a new method based on IEEE802.15.4 with wireless sensor networking (WSN) for advanced healthcare monitoring using two techniques. First, wearable healthcare devices located in the human body. Second, a number of sensors placed in house celling with all information transmitted to the server in order to be stored and later analysed. The key important point behind applied like this system is to provide periodic monitoring system when situation required. Our literature review shows the current contributions are still limited for providing 
immediate information about SCD normality or abnormality [13], [15]. Therefore, this research work was focusing on the field of building robust system that can track disease and send instant information to the medical consultants in order to provide accurate decision, especially with critical conditions cases.

\section{System Design of Healthcare Based on Mobile Healthcare (M- healthcare)}

In this research, we present a framework for applying smartphone device management system for the ubiquitous healthcare. The proposed system consists of mobile Platform, network coordinator and personal computers. In our framework, there are two important sides that have been proposed. The patient side is responsible for analysis, data storing and send feedback messages to the healthcare professionals in association with high-risk condition. The high-risk condition can be obtained when the number of heartbeat is significantly increasing or breathing becomes difficult also known as vaso-occlusive crisis. The medical side is responsible for providing accurate decision support to advice patient by taking appropriate type of medicine, particularly within crisis cases. Therefore, the proposed system has the ability of analysing and storing data without sending the information to the health professionals when detecting low-level condition with regards to reduce communication cost, workload, and encourage personalised patient's self-care system. The low-level condition can be obtained when the number of heartbeat is slightly decreasing. The proposed framework design is shown in Figure 1.

The deployment of self-care management system consists of two main categories, the data collecting, and data analysis. In this paper, we also consider medical centre (located at hospital and clinics environments). The overall responsibility of medical centre is monitoring patients, gather data and sending reminder when the data indicates the patient is in critical condition. 


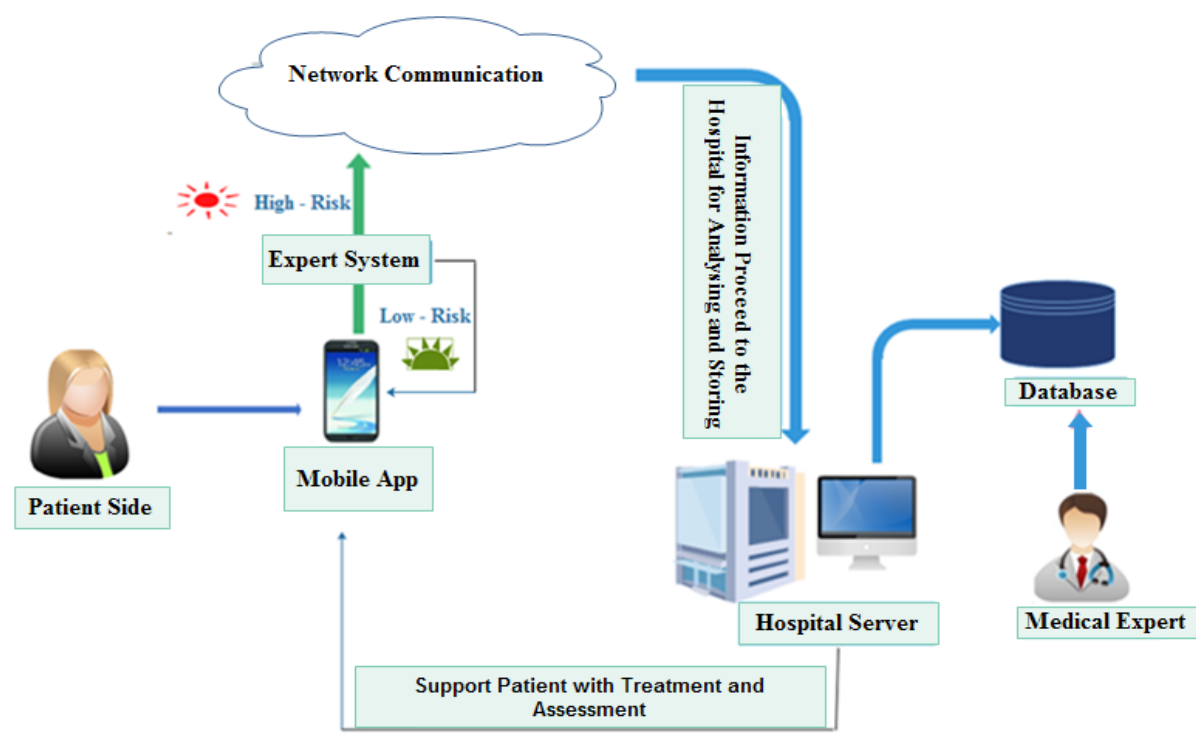

Fig.1. System architecture of the proposed system.

These days, smart devices play such an important role to support people by accessing their condition from home. Within this context, mobile technology were used in this research to analyse all data that collected from patient's body in order to send that data to the medical consultants for diagnosing the patient's situation. The communications platform between patient and mobile device will be implemented through the application interface. In this aspect, we intend to store all the relevant information that come through patients input with the purpose of further investigations and analysis. It is essential that a patient who is suffering from SCD, is required to store relevant information, for instance personal details. This information can be used in the processing stage, which will be located in the device's platform. This would in turn, remind the patient of their current condition. Furthermore, the system then carries out analysis on the data that sent to determine whether the patient's condition is normal or abnormal. Afterward, the system will advise the patient of what medication is needed to correct their condition promptly and accurately. Otherwise, the system needs to send the whole details to a medical consultant which in turn to decide what action is required in order to save patient's life. The main motivation behind utilising mobile application (app) is to determine the patients' condition more promptly.

Based on the large volume of data that is generated in medical sectors, it has become vital to utilise expert system in the purpose of enhancing the quality of care. Expert system provides an effective and reliable way on improving the patients facilities within healthcare sectors [14]. The main significant factors that we used 
expert system in this paper is to deliver unlimited services. For example, managing, analysing and diagnosing patient's data to detect normal and abnormal patterns in order to save the patient's life.

\subsection{Self-Care Management System}

There is no laboratory facilities available to test the blood for genetic blood disorder in the patients' home. The main reason behind this is because of the high financial costs of installing a blood test machine in the patient's home. In addition, the blood test machine requires a specialist nurse or medical expert to understand the main attributes that will be obtained after the blood has been tested. There are four types of SCD which is considered of an abnormal level, for instance (Hb SS, Hb SC, Hemoglobin S Beta + Thalassemia, Hemoglobin S Beta +0 Thalassemia). The patient's diagnosis will be assessed according to the symptoms (e.g. severe pain in the bones, painful enlargement of the spleen and heart problems, headache, very pale skin, and chest pain) that could appear in various areas of the body. The data will be collected by the android mobile App, which will provide patients with a set of multi choice to select the proper symptoms. The proposed expert system will then analyse the input data and determine whether the patient's condition is normal or abnormal. This information will be transferred to the medical center in order to be analysed by a professional who makes any decisions needed in order to tackle patient's condition. This would be extremely beneficial, within crisis cases. In these circumstances, the healthcare professionals or specialist nurses could contact patients for professional advises on their condition.

\section{Methodology}

This paper has proposed a framework for monitoring and diagnosis SCD for the purpose of helping patients and healthcare organisation. The first component of the proposed system, is the classification of the sickle cell patients from normal patients using machine learning algorithms.

The original dataset was collected from a local hospital in Liverpool, UK for the purpose of classifying normal and sickle cell patients. Within this context, the local hospital has been selected according to the highest number of patients who actually suffer from SCD. The data represents 12 primary attributes using 100 patients checked for SCD of which 63 tested negative (patients has already carrying SCD traits), whereas 37 person tested positive (Non SCD traits affected). Table 1 illustrates the characteristics of SCD. 
Table 1. Characteristics of SCD dataset.

\begin{tabular}{|c|l|l|l|}
\hline No & $\begin{array}{l}\text { Types of } \\
\text { Attributes }\end{array}$ & \multicolumn{1}{c|}{ Min } & \multicolumn{1}{c|}{ Max } \\
\hline 1 & Gender & Male, Female & Male, Female \\
\hline 2 & Age & Between 15 & Between 80 \\
\hline 3 & Hb & g/L 118 & g/L 148 \\
\hline 4 & Hb F & $0.8 \%$ & $2.0 \%$ \\
\hline 5 & PLTS & x109/L 150 & x109/L 400 \\
\hline 6 & MCV & fL 80 & $\mathrm{fL} 100$ \\
\hline 7 & BILI & umol/L 21 & umol/L 30 \\
\hline 8 & ALT & U/L 35 & U/L 45 \\
\hline 9 & EGFR & $\mathrm{ml} / \mathrm{min} / 1.73 \mathrm{~m} 2>45$ & $\mathrm{ml} / \mathrm{min} / 1.73 \mathrm{~m} 2>60$ \\
\hline 10 & Ferritin & ug/113 & ug/150 \\
\hline 11 & Urea & $\mathrm{mmol} / \mathrm{L} 2.5$ & $\mathrm{mmol} / \mathrm{L} 7.8$ \\
\hline 12 & creatinine & umol/L 50 & umol/L 130 \\
\hline
\end{tabular}

\subsection{Confusion Matrix Evaluation}

In this section, there are two individual classification in the field of machine learning (including Zero-Rule and Instance-based learning algorithm) have examined to find the better value for Accuracy by class and the weight average. The evaluation technique was carried out in a confusion matrix (also known as contingency table). Figure 2 illustrates the confusion matrix. There are four denotes that are located in the contingency table. True Negative (TN) and True Positive (TP) donates are considered one of the most accurate classification of negative instance and the accurate classification of positive instance respectively. In addition, False Negatives (FN) illustrate the positive instance, which is incorrectly classified in terms of negative type, whereas False Positives (FP) show negative symbols, which is incorrectly classified in association with positive type.

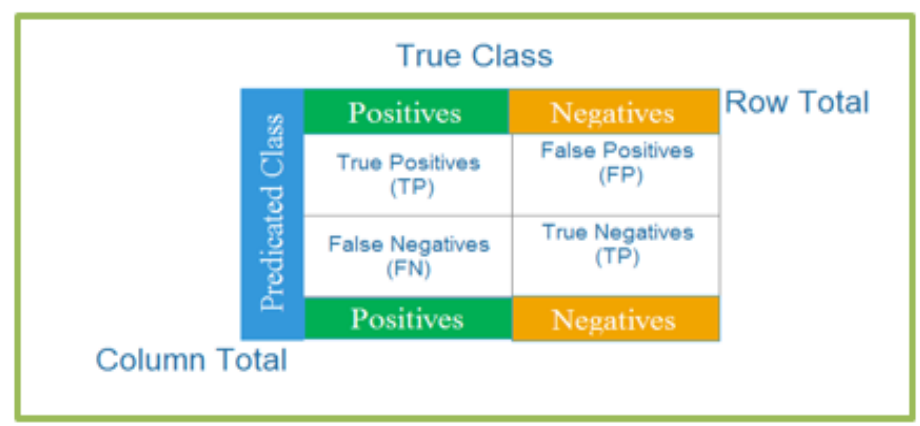

Fig. 2. Confusion matrix. 
Based on the confusion matrix, there are a number of measurements that can be acquired to examine the model performance in terms of accuracy (also known as producer's accuracy) this can be determined using formula (1) below.

$$
A C=\frac{T P+T N}{(T P+F P)+(F N+T N)}
$$

The main purpose of applying equation (2) is to evaluate the proportion of positive instances that were correct.

$$
T P=\frac{T N}{(F N+T N)}
$$

The FP were classified incorrectly can be obtained from the following equation that illustrated in (3).

$$
F P=\frac{T N}{(T P+F P)}
$$

In order to classify the $\mathrm{TN}$ that were classified correctly, equation (4) were conducted.

$$
T N=\frac{T P}{(T P+F P)}
$$

The FN is belong positives instances were incorrectly classified. The following formula (5) illustrates how datasets can be calculated.

$$
F N=\frac{F N}{(F N+T N)}
$$

To evaluate the accuracy, it is typical practice to utilise confusion matrix. In this case, the confusion matrix mainly depends on the selection of datasets. Our motivation behind applying contingency table is to improve the accuracy and performance of benchmark datasets.

\subsection{Data Collection}

The first primary aspect is how to collect data for SCD patients. In order to collect SCD dataset, a blood test machine is required in the hospital side. Unfortunately, due to the financial cost of monitoring requirements needed by the patient, medical consultants were not be able to meet with the patient's demands as well as maintain the required standard of medical facility; this puts a lot of strain on consultants in which the total cost is almost un-affordable. An intelligent system becomes a very significant technique to analyse the huge amount of datasets that assist healthcare professionals for a better understanding. The gathered data will be represented in the best possible method based on machine learning algorithm.

This research proposed Zero-Rule based and Instance-based learning algorithm for diagnosing and managing SCD. Data was simulated using real-life data sets provided 
by local hospital when a patient takes blood test sample, which contains a set of attributes that used for checking patients' condition. In the medical centre, there are 12 attributes were used in this research as shown in Table 1 (e.g., Haemoglobin (Hb), Platelets (PLTS), Mean Corpuscular Volume (MCV), Neutrophils (white blood cell NEUT), Reticulocyte Count (RETIC), Reticulocyte Count (RETIC F), Body Bio Blood (BIO), Hb F, Bilirubin (BILI), Alanine aminotransferase (ALT), an Aspartate Aminotransferase (AST), Lactate dehydrogenase (LDH)). Furthermore, there are two features should be considered when analysing the blood test, which are gender and age. These features have some significant effects on the blood test.

The Zero Rule algorithm with cross validation was utilised for the classification of the patients' data. It is a rule based classifier which is also called 0-R. Table 2 shows the accuracy and average weight classified by the proposed algorithm. The correct classified Instances that we obtained after utilising Zero-Rule algorithm is 62\%, whereas, incorrect classified Instances is $38 \%$. Further accuracy of the classifier is shown in Table 3.

Table 2. Classifier output for Zero-Rule algorithm.

\begin{tabular}{|c|c|c|}
\hline No & Features & $\begin{array}{c}\text { Classifier } \\
\text { output }\end{array}$ \\
\hline 1 & Correctly Classified Instances & $62 \%$ \\
\hline 2 & Incorrectly Classified Instances & $38 \%$ \\
\hline 3 & Kappa statistic & 0 \\
\hline 4 & Mean absolute error & 0.4722 \\
\hline 5 & Root mean squared error & 0.4858 \\
\hline 6 & Relative absolute error & $100 \%$ \\
\hline 7 & Root relative squared error & $100 \%$ \\
\hline 8 & Total Number of Instances & 100 \\
\hline
\end{tabular}

Table 3. Accuracy by class for Zero-Rule algorithm.

\begin{tabular}{|c|c|c|}
\hline Measures & $\begin{array}{c}\text { Accuracy By } \\
\text { Class }\end{array}$ & Weighted Avg. \\
\hline True Positive Rate & 1 & 0.62 \\
\hline $\begin{array}{c}\text { False Positive } \\
\text { Rate }\end{array}$ & 1 & 0.62 \\
\hline Precision & 0.61 & 0.372 \\
\hline Recall & 1 & 0.61 \\
\hline F-Measure & 0.758 & 0.462 \\
\hline ROC Area & 0.483 & 0.483 \\
\hline
\end{tabular}


In order to enhance our result with more achievable outcomes, we have used another simple classifier which is called the Instance-based learning algorithm developed by Aha et al. [15]. The outcomes with proposed classifier shows better performance than Zero-Rule algorithm. A performance of $99 \%$ accuracy was achieved as illustrated in Tables 4 and 5.

Table 4. Classifier output for the Instance-based learning algorithm.

\begin{tabular}{|c|c|c|}
\hline No & Features & Classifier output \\
\hline 1 & Correctly Classified Instances & $99 \%$ \\
\hline 2 & Incorrectly Classified Instances & $1 \%$ \\
\hline 3 & Kappa statistic & 0.9789 \\
\hline 4 & Mean absolute error & 0.01 \\
\hline 5 & Root mean squared error & 0.1 \\
\hline 6 & Relative absolute error & $2.1179 \%$ \\
\hline 7 & Root relative squared error & $20.5858 \%$ \\
\hline 8 & Total Number of Instances & 100 Patients \\
\hline
\end{tabular}

Table 5. Accuracy by class for Instance-based learning algorithm.

\begin{tabular}{|c|c|c|}
\hline Measures & $\begin{array}{c}\text { Accuracy By } \\
\text { Class }\end{array}$ & Weighted Avg. \\
\hline True Positive Rate & 1 & 0.99 \\
\hline $\begin{array}{c}\text { False Positive } \\
\text { Rate }\end{array}$ & 0.016 & 0.006 \\
\hline Precision & 0.974 & 0.99 \\
\hline Recall & 1 & 0.99 \\
\hline F-Measure & 0.987 & 0.99 \\
\hline ROC Area & 0.992 & 0.992 \\
\hline
\end{tabular}

The Instance-based learning algorithm achieved a better outcomes in comparison to Zero-Rule algorithm due to the optimal accessible heuristic for calculating the values of attributes. This algorithm can deal with large amount of datasets that need to be classified. In addition, this technique requires low storage and provides high performance in terms of classification accuracies. Our motivation behind using Instance-based learning algorithm is that it can save documents of completed trainingset. Then, compares any new documents to be classified with the saved data. Furthermore, it can classify a large number of new datasets more rapidly. The performance method compare among other techniques are considerably much more beneficial in providing accurate outcomes. Thus, the main purpose of applying these two experiments is to examine all the attributes (excluding the class value) whether the patients carry SCD traits or otherwise. 


\section{Conclusion}

This paper proposed a new framework for self-care management system in order to improve the idea of electronic healthcare for supporting patient's medical facilities. This will provide better satisfaction, quality of service, and lowering the costs of patient care. Self-care monitoring system is the most crucial function for patients' daily life. This could provide the ability to check and monitor their own health condition through smart devices technology. Based on this technology, personal monitoring system supports SCD patients to manage their health status instead of visiting healthcare professionals at regular time or being admitted into hospitals. Two machine learning algorithms were used to classify patients with SCD. The Instancebased learning algorithm offers an important improvement in terms of accuracy over Zero-Rule classifier with 99\% accuracy in comparison to 62\% using Zero-Rule algorithm. In this case, the possible way to improve the accuracy of these experiments is to increase the sample size of the datasets.

\section{References}

[1] D. J. Weatherall, "The Role of the Inherited Disorders of Hemoglobin, the First "Molecular Diseases," in the Future of Human Genetics," Annual review of genomics and human genetics, vol. 14, pp. 1-24, 2013.

[2] D. J. Weatherall, "The importance of micromapping the gene frequencies for the common inherited disorders of haemoglobin," British journal of haematology, vol. 149, pp. 635-637, 2010.

[3] D. J. Weatherall, "The inherited diseases of hemoglobin are an emerging global health burden," Blood, vol. 115, pp. 4331-4336, 2010.

[4] M. K. Lin, "Evaluating the acceptance of mobile technology in healthcare: development of a prototype mobile ECG decision support system for monitoring cardiac patients remotely," University of Southern Queensland, 2012.

[5] G. Gillespie, "Deploying an IT cure for chronic diseases," Health data management, vol. 8, p. 68, 2000.

[6] V. Chan, P. Ray, and N. Parameswaran, "Mobile e-Health monitoring: an agent-based approach," IET communications, vol. 2, pp. 223-230, 2008.

[7] R. Tabish, A. M. Ghaleb, R. Hussein, F. Touati, A. Ben Mnaouer, L. Khriji, et al., "A 3G/WiFi-enabled 6LoWPAN-based U-healthcare system for ubiquitous real-time monitoring and data logging," in Biomedical Engineering (MECBME), 2014 Middle East Conference on, 2014, pp. 277280.

[8] T. W. Kim, K. H. Park, S. H. Yi, and H. C. Kim, "A Big Data Framework for u-Healthcare Systems Utilizing Vital Signs," in Computer, Consumer and Control (IS3C), 2014 International Symposium on, 2014, pp. 494-497. 
[9] P. Leijdekkers and V. Gay, "Personal heart monitoring and rehabilitation system using smart phones," in Mobile Business, 2006. ICMB'06. International Conference on, 2006, pp. 29-29.

[10] K.-R. Chen, Y.-L. Lin, and M.-S. Huang, "A mobile biomedical device by novel antenna technology for cloud computing resource toward pervasive healthcare," in Bioinformatics and Bioengineering (bibe), 2011 ieee 11th International Conference on, 2011, pp. 133-136.

[11] S. Yang, E. Jacob, and M. Gerla, "Web-based mobile e-Diary for youth with Sickle Cell Disease," in Consumer Communications and Networking Conference (CCNC), 2012 IEEE, 2012, pp. 385-389.

[12] J. Venugopalan, C. Brown, C. Cheng, T. H. Stokes, and M. D. Wang, "Activity and school attendance monitoring system for adolescents with sickle cell disease," in Engineering in Medicine and Biology Society (EMBC), 2012 Annual International Conference of the IEEE, 2012, pp. 2456-2459.

[13] S.-J. Jung, T.-H. Kwon, and W.-Y. Chung, "A new approach to design ambient sensor network for real time healthcare monitoring system," in Sensors, 2009 IEEE, 2009, pp. 576-580.

[14] J. A. Doherty, R. M. Reichley, L. A. Noirot, E. Resetar, M. R. Hodge, R. D. Sutter, et al., "Monitoring pharmacy expert system performance using statistical process control methodology," in AMIA Annual Symposium Proceedings, 2003, p. 205.

[15] D. W. Aha, D. Kibler, and M. K. Albert, "Instance-based learning algorithms," Machine learning, vol. 6, pp. 37-66, 1991. 